\title{
Enhancing power density of biophotovoltaics by decou- pling storage and power delivery
}

Kadi L. Saar ${ }^{1}$, Paolo Bombelli ${ }^{1,2}$, David J. Lea-Smith ${ }^{2,3}$, Toby Call ${ }^{2}$, Eva-Mari Aro ${ }^{4}$, Thomas Müller $^{1,5}$, Christopher J. Howe ${ }^{2} \&$ Tuomas P. J. Knowles ${ }^{1,6}$

${ }^{1}$ Department of Chemistry, University of Cambridge, Lensfield Road, Cambridge CB2 1EW, UK

${ }^{2}$ Department of Biochemistry, University of Cambridge, Tennis Court Road, Cambridge CB2 $1 Q W, U K$

${ }^{3}$ Current address: School of Biological Sciences, University of East Anglia, Norwich Research Park, Norwich NR4 7TJ, UK

${ }^{4}$ Laboratory of Molecular Plant Biology, Department of Biochemistry, University of Turku, Turku, FI-20014, Finland

${ }^{5}$ Fluidic Analytics Limited, Cambridge, UK

${ }^{6}$ Cavendish Laboratory, Department of Physics, University of Cambridge, J J Thomson Ave, Cambridge $C B 3$ OHE, $U K$

Biophotovoltaic devices (BPVs), which use photosynthetic organisms as active materials to harvest light, have a range of attractive features relative to synthetic and non-biological photovoltaics, including their environmentally friendly nature and ability to self-repair. However, efficiencies of BPVs are currently lower than those of synthetic analogues. Here, we demonstrate BPVs delivering anodic power densities of over $0.5 \mathrm{~W} \mathrm{~m}^{-2}$, a value five-fold higher than for previously described BPVs. We achieved this through the use of cyanobacte- 
rial mutants with increased electron export characteristics together with a microscale flowbased design that allowed independent optimisation of the charging and power delivery processes, as well as membrane-free operation by exploiting laminar flow to separate the catholyte and anolyte streams. These results suggest that miniaturisation of active elements and flow control for decoupled operation and independent optimisation of the core processes involved in BPV design are effective strategies for enhancing power output and thus the potential of BPVs as viable systems for sustainable energy generation.

Energy demand driven by a rising global population must increasingly be satisfied from renewable alternatives to fossil fuels, as the latter release extensive amounts of greenhouse gases with potentially devastating consequences on our ecosystem. Solar power is considered to be a particularly attractive source as on average the Earth receives around 10,000 times more energy from the sun in a given time than is required by human consumption ${ }^{1,2}$. Although a number of technologies exist to convert this extensively available sunlight into electrical current ${ }^{3,4}$, factors such as scarcity of production materials, high cost per delivered quantity of electricity, and lack of equally efficient storage technologies have limited their adoption ${ }^{4-8}$. Biological photovoltaics (BPVs; also known as biophotovoltaics and biological solar-cells ${ }^{9}$ ) are emerging as an environmentally friendly and low-cost approach to harvest solar energy and convert it into electrical current ${ }^{10-12}$. In phototrophic organisms, light is converted into high-energy charge-separated electron-hole pairs and the excited electrons are transferred through a number of intracellular electron carriers, with a fraction eventually exported across the cell membrane and released to the external environment ${ }^{13-15}$ (Figure 1a). 
In BPVs these secreted electrons are directed to an electrode (anode) and from there allowed to flow to a more positive potential electrode (cathode) through an external circuit, thus generating current $^{16,17}$. Simultaneously, the protons released by the cells diffuse from the anodic chamber to the cathodic one where water is re-formed on an appropriate catalyst (Figure 1b). This process leads to the generation of current without release of any chemical side products. A proton permeable membrane separates the anodic chamber from the cathodic one ensuring that electrons travel only via the external load.

BPVs demonstrated to date rely either on suspending photosynthetic cells in solution or immobilising them directly onto the anode ${ }^{9}$. In these designs, electron generation and transfer to the electrical circuit occur in a single compartment, and the electrons reach the anode and generate current as soon as they have been secreted. Here, we propose a two chamber system where charging (reduction of the electron carrier molecules by exoelectrogenic electrons) and power delivery (electron transfer to the external electrical circuit) are spatially decoupled from one another (Figure 1). In such a system it becomes possible to design the geometrical configurations and operating conditions of the electron generation and power harvesting units independently, which allows their performance to be optimised simultaneously. In particular, in the devices described here, we exploit miniaturised geometries in the power delivery unit. This operation at small length scales suppresses convective mixing ${ }^{18}$, enabling us to omit the semipermeable membrane from the power delivery unit of the BPV, normally required to separate the device into the anodic and cathodic compartments. In addition to decreasing the internal resistance of the device, omitting the membrane would reduce the cost of the system and make the operation easier as membranes have been 


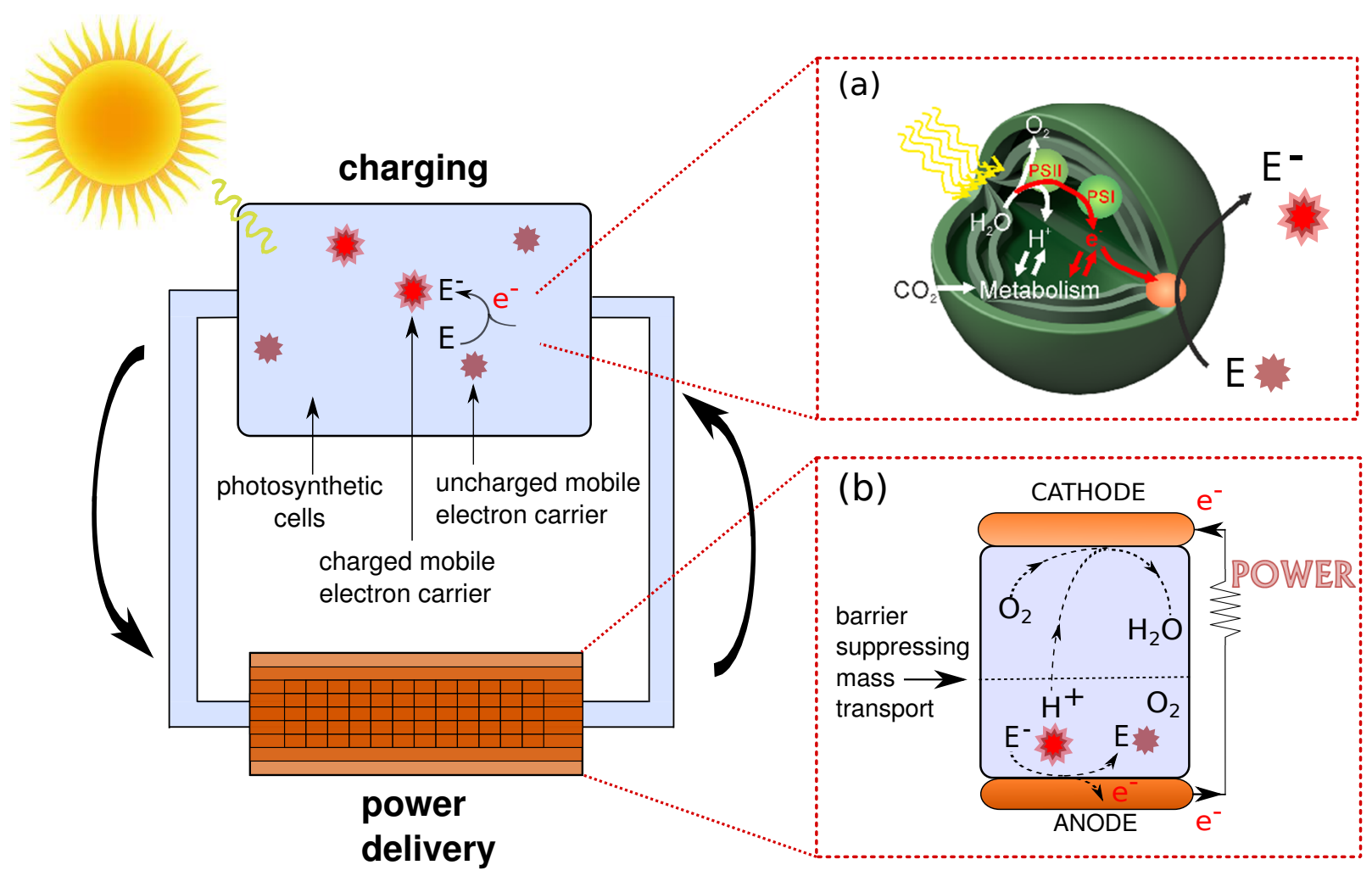

Figure 1: The charging and delivery processes in a flow-BPV. The two processes can be spatially decoupled, providing temporal flexibility over power generation and enabling their independent optimisation. Inset (a): In the charging unit high energy electrons $\left(\mathrm{e}^{-}\right)$are generated by the photosynthetic cells using photosystems I and II (PSI, PSII), and some are released to the external environment via the exoelectrogenic activity of the cells to reduce the charge carriers $\left(\mathrm{E} \rightarrow \mathrm{E}^{-}\right)$. Inset (b): In the power delivery unit the reduced charged carriers $\left(\mathrm{E}^{-}\right)$are brought into contact with the anode from where the electrons flow to the cathode, generating current. Protons $\left(\mathrm{H}^{+}\right)$diffuse to the cathode where water is catalytically re-generated while the electrons reach the cathode only via the external circuit due to controlled mass transport at small length scales. 
reported to dry out, degrade, foul and $\operatorname{cog}^{19-21}$. More generally, the use of small length scales has the potential to decrease the resistive electrical losses of the system due to elevated surface-tovolume ratios, enhanced mass transfer coefficients and small electrode separation ${ }^{20-23}$.

We show that the use of such two chamber flow-controlled BPVs resulted in a two and a half fold increase in anodic power density compared to the maximum reported so far when using wild-type Synechocystis sp. PCC6803 cells as the phototrophic catalyst (Supplementary Table 1). When replacing these with Synechocystis mutant cells deficient in the main photosynthetic and respiratory electron sinks, the terminal oxidases and the flavodiiron complexes Flv1/3 and Flv2/4, the improvement in the power density further increased to five-fold ${ }^{24,25}$.

\section{Device design and operation}

The power delivery unit of the BPV was fabricated in poly(dimethylsiloxane) via soft photolithography as a rectangular channel with one inlet for the anodic and one for the cathodic fluid (Figure 2a; Methods). Due to the similar viscosities of the anolyte and the catholyte solutions, the boundary between the two half-cells was determined by the relative flow rates of the two fluids ${ }^{26,27}$. Specifically, it was set at a distance of $20 \%$ of the total width of the device away from the anode, so that, due to the limited residence time of the fluids in the device, a significantly larger fraction of the electron carrier ions would reach the anode than the cathode by molecular diffusion. Furthermore, the dimensions of the system were chosen such that protons, which have over an order of magnitude higher diffusion coefficient than the electron carrier ions, diffuse to the cathode 
within the limited residence time of the fluids in the device while the electron carrier molecules remain in the vicinity of the anode. This generates an effective diffusion-controlled proton permeable barrier between the cathodic and anodic areas (Figure 2a, inset (ii)) which only protons can cross (Figure 2a, inset (iii)). The side-wall electrodes filled the full height of the device, with the anode spanning its full length and the cathode being a point electrode at the end of the device (see Methods).

We used Synechocystis sp. PCC6803 cells, motivated by their previously demonstrated exoelectrogenic activity ${ }^{16,17}$ and the availability of genetic tools to allow generation of multiple mutations in genes for electron transfer components ${ }^{28}$. The cells were pre-mixed with the electron carrier $\mathrm{K}_{3}\left[\mathrm{Fe}(\mathrm{CN})_{6}\right]$ to facilitate the transfer of the exoelectrogenic electrons to the anode. This electron carrier has previously been shown to be physiologically well tolerated by Synechocystis sp. PCC6803 over extended time periods at the concentration used in this study ${ }^{17}$. The mixture was left for 3 hours for the electrons released by the photosynthetic cells to reduce the electron carrier ions, after which the fluids were injected to the device and the current-voltage (polarisation) curve was acquired by varying the applied voltage and recording the respective current (Methods). Unlike approaches that change the resistor connected to the system and hence vary the voltage and the current simultaneously, potentiometric measurements alter only one parameter and have become a commonly used method for BPV characterisation ${ }^{29,30}$. We further collected the anoyte and catholyte solutions at the device outlets, and after illuminating the cell suspension for an additional 3 hours to recharge the electron carrier molecules the solutions were re-injected to the device. Crucially, the device performance was unaffected between the two rounds of recirculation (Figure $2 \mathrm{~b}$ ), 
(a)

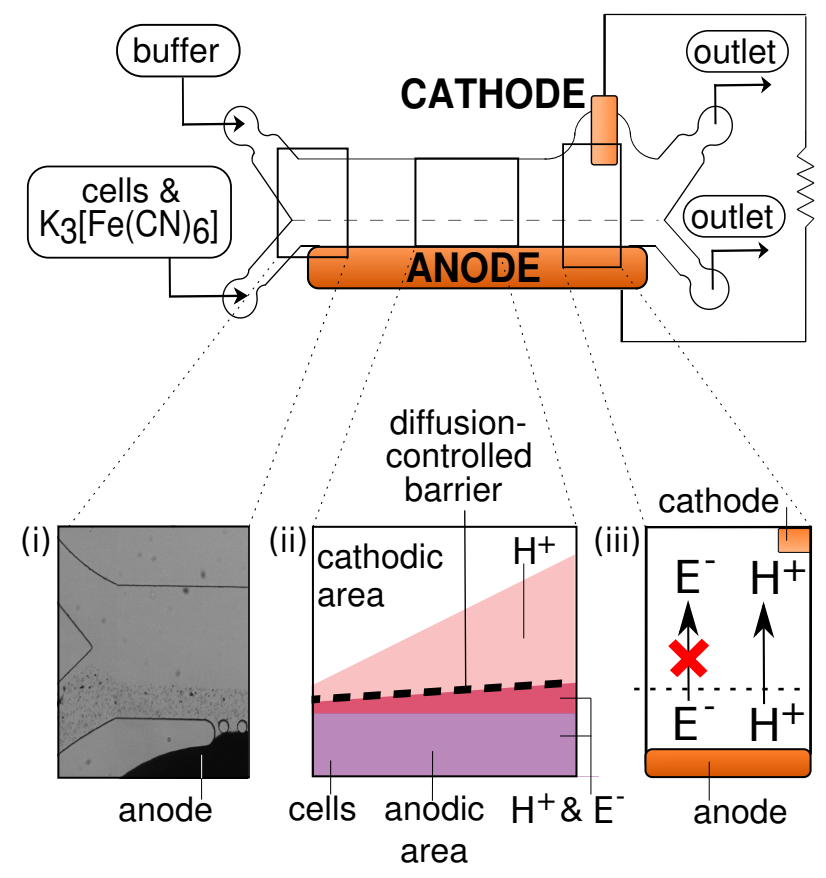

(b)

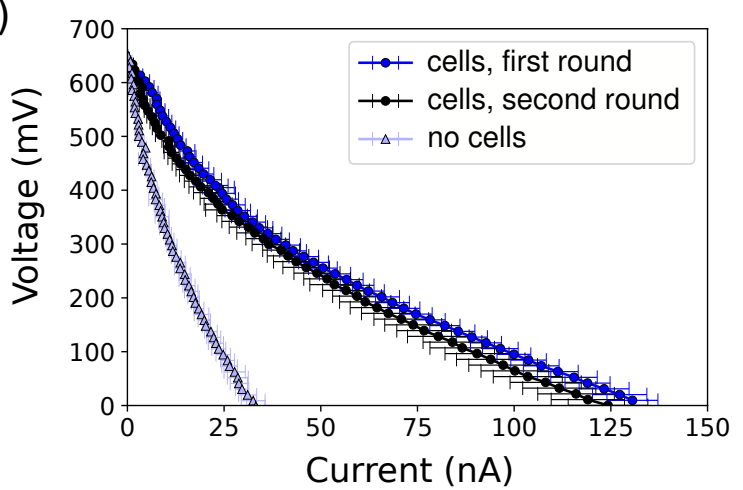

(c)

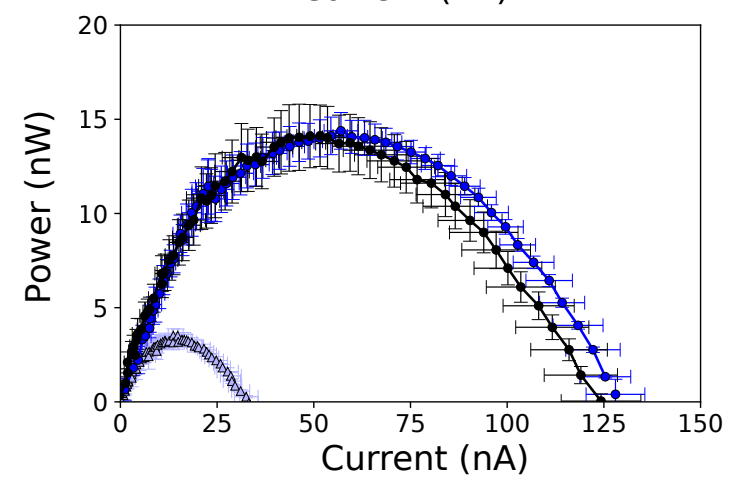

Figure 2: Construction and performance of the micro-channel BPV ( $\mu$-BPV). (a) Schematic diagram (not to scale) of the power delivery unit of the $\mu$-BPV. Green circles represent cyanobacterial cells. Inset (i) shows the input channels. Due to negligible inertial forces flow profiles in the channels are laminar which generates a diffusion-controlled barrier (inset (ii); purple colour indicates the position of protons and electron carrier ions if there were no mass transport between the cathodic and anodic streams, light and dark pink colours the areas where protons and electron carriers can diffuse to, respectively). This barrier can be crossed only by fast diffusing species, in this case protons (inset (iii)). (b) The polarisation and (c) power curves for the fabricated $\mu$-BPV at chlorophyll concentration of $c_{c h l}=8 \mu M$ and flow rate of $Q_{\text {total }}=20 \mu \mathrm{L} \mathrm{h}^{-1}$ on the first (blue circles; average of $n=3$ repeats; error bars correspond to standard deviations) and second injection (black circles) and without cells (light blue triangles). 
indicating the pressure drops of ca. $100 \mathrm{~Pa}$ inherent in the device operation (Supplementary Note 1) did not damage the cells or affect their exoelectrogenic ability.

We noted that whereas the $\left[\mathrm{Fe}(\mathrm{CN})_{6}\right]^{-4}$ produced by the electrons released by the photosynthetic cells oxidised slowly back to $\left[\mathrm{Fe}(\mathrm{CN})_{6}\right]^{-3}$, the rate of this reaction - measured to be around $5 \mathrm{nM} \mathrm{s}^{-1}$ (Supplementary Figure 1a) - is significantly lower than that of the $\left[\mathrm{Fe}(\mathrm{CN})_{6}\right]^{-4}$ generation by the electrons released by the cells $\left(280 \mathrm{nM} \mathrm{s}^{-1}\right.$; Supplementary Figure 1b). Although eliminating oxygen could potentially prevent any back-oxidation, the challenges associated with its removal are unlikely to outweigh the potential gain, especially as oxygen removal could also affect the viability of the cells.

From the measured polarisation and power curves (Figure $2 b, c)$, we deduced the internal resistance of the BPV to be approximately $3 \mathrm{M} \Omega$ and the resistivity of the device to be around $200 \Omega \mathrm{m}$; due to the varying area between the anode (spanning the full length of the device) and the cathode (a point electrode) the latter value is an approximation and for the purpose of the current estimate was obtained by using the average of the two areas. This resistivity is substantially smaller than for our previously built devices of larger scale $-19 \mathrm{k} \Omega \mathrm{m}^{17}$ and $69 \mathrm{k} \Omega \mathrm{m}^{12}$, both of which had similarly used BG-11 as the carrier medium. This decrease is most likely to originate from the reduced resistive losses at the smaller length scales and from the absence of a semipermeable membrane in the design of the BPV ${ }^{20-23}$. Additionally, we did not observe a tendency for the polarisation curves to show higher gradients, even at high currents. This finding indicates that the performance of the device was not limited by diffusion or mass transfer ${ }^{22,29}$. 
Small background currents were recorded when the device was operated with an anolyte including only the buffer and the electron carrier with no cyanobacterial cells present. Such background currents have been reported previously and attributed to the ionic concentration of the medium and slow formation of electrolysis products at the anode ${ }^{31,32}$. This background current was similar between all the devices used. As in previous studies, the peak biotic power of each device was taken as the difference between the peak power of the device operated with cyanobacterial cells and the device operated without the cells at the same current ${ }^{9,32}$. The average peak biotic power output for the micro-channel BPV ( $\mu$-BPVs) was recorded to be $13.9 \pm 0.9 \mathrm{nW}$ (Supplementary Figure $2 ; n=3$ repeats in three individually fabricated devices, error bars correspond to standard deviations) at a current of $60 \mathrm{nA}$.

\section{$\mu$-BPV performance}

We expect the performance of the flow $\mu$-BPV to depend on the flow rates of the fluids into the anodic and the cathodic half-cells. Specifically, at higher flow rates not all the charged electron carriers can diffuse to the anode within their residence time in the device. By contrast, at low flow rates, the ions of the reduced electron carrier can also cross the channel and lead to a shortcircuit in the device. Analogous behaviour has been reported for microbial fuel cells as the fuel cross-over concept $^{33,34}$, and the termination of flow has been observed to suppress power output entirely ${ }^{35}$.

The effect of the flow rate on the BPV performance was investigated by keeping the ratio of the

cathloyte and anolyte flow rates fixed $\left(\frac{Q_{\text {cathodic }}}{Q_{\text {anodic }}}=4\right)$ and recording the power curves at a range 
of total flow rates. The peak biotic power outputs were then normalised to the flow rate of the fluids into the device which in turn was proportional to the number of charge carriers that entered the device in a given time. This normalised power output was observed to decrease with the flow rate (Figure 3a; blue circles, average of $n=3$ repeats) and can be explained by the less efficient use of the electron carrier ions at higher flow rates. The absence of such a decrease at low flow rates, $O\left(10 \mu \mathrm{L} \mathrm{h}^{-1}\right)$ is likely to be due to the effect of a more significant cross-over of the charged electron carriers at the smaller flow rate, complete reduction of the electron carrier or both. We further simulated ${ }^{36}$ the diffusion of the electron carrier ions in the rectangular channel to estimate the fraction of ions that can diffuse to the two electrodes as a function of flow rate. The experimental data for the normalised power were observed to follow a similar trend to the simulations but the recorded power output declined faster than the fraction of electron carriers that reached the anode (Figure 3a; black line). It is likely that under these conditions further factors limit the performance of the device, including the availability of protons on the cathode or mass transfer limitations at the surface of the anode.

A significant difference of a flow-based system in comparison to those that rely on biofilms grown on the anode is the requirement to drive fluid flow within the device. To recirculate the fluids continuously, they have to be pumped back to higher pressures before re-entering the device (Figure 1). Higher flow rates lead not only to more inefficient use of the fuel (Figure 3a) but also to increased frictional losses (Supplementary Note 1, equation(1)). At low flow rates, the power output of our demonstrated flow-BPV more than compensated for the energy losses from the viscous drag even when accounting for non-ideal operation of pumps (Figure 3b; typical efficiencies vary from 65\% 
(a)

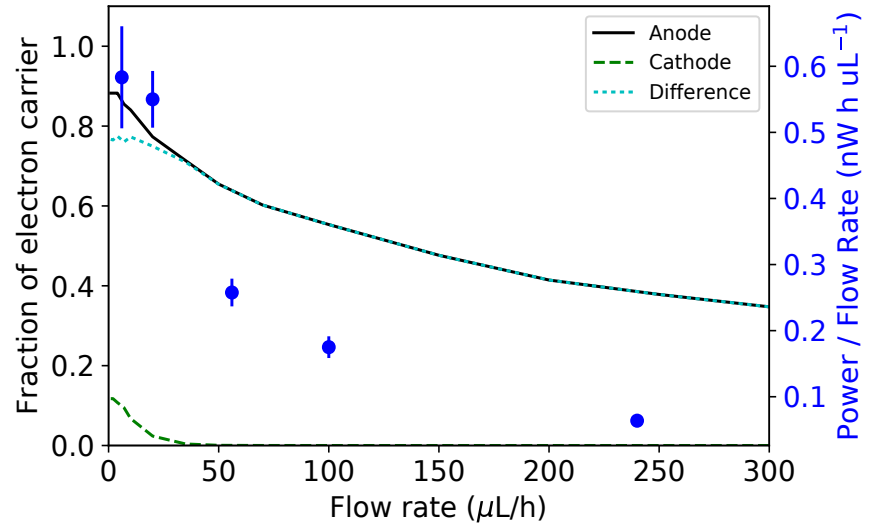

(b)

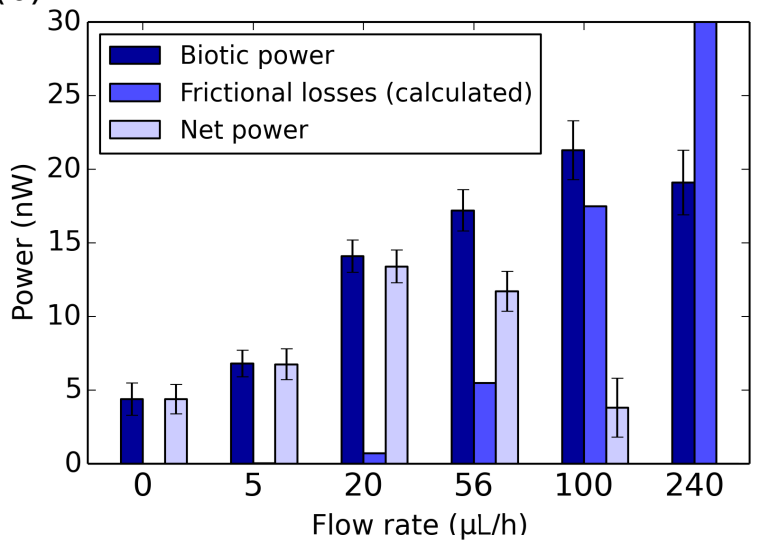

Figure 3: The effect of flow rate on the device performance. (a) The biotic power outputs normalised by flow rate (blue circles, average of $n=3$ repeats with error bars corresponding to standard deviations; $\frac{Q_{\text {cathodic }}}{Q_{\text {anodic }}}=4$ for all) decreased with flow rate. One of the factors contributing towards this decrease is the reduced number of electron carrier ions reaching the anode (solid line). (b) The delivered biotic power, power loss due to friction and the net power output at different flow rates (error bars are standard deviations for $n=3$ measurements). $Q_{\text {total }}=20 \mu \mathrm{L} \mathrm{h}^{-1}$ was chosen for further experiments due to the highest net power output being recorded at this value.

to $90 \%$ ). However, comparing the performance of the device at various flow rates, we concluded that the increase in the biotic power output at higher flow rates is eventually counterbalanced by the elevated power consumption needed to overcome the frictional losses. Thus the optimal flow rate maximising the overall power output was found to be around $20 \mu \mathrm{L} \mathrm{h}^{-1}$ (Figure 3b) and was therefore used for all further experiments. 


\section{Multiparameter optimisation of the power output of the $\mu-\mathrm{BPV}$}

Unlike devices where photosynthetic cells transfer their electrons directly to the anode, we used a design strategy where the charging and the power delivery processes are spatially decoupled. Combined with the use of mobile electron carriers that are free to move and collect electrons from all the cells, this strategy has the potential to lead to elevated current outputs when increasing the concentration of cells under otherwise identical conditions. We set out to test this hypothesis by characterising the performance of the device at two different concentrations of cells. We found that the peak power output increased with the cell concentration reaching $(22.2 \pm 1.5) \mathrm{nW}$ (average of $n=3$ repeats) at chlorophyll concentration of $40 \mu \mathrm{M}$ (Figure $4 \mathrm{a}$ ). We observed that the device

yielded even larger power outputs at higher cell concentrations and in particular, recorded the peak power output to be $65 \mathrm{nW}$ at $\mathrm{c}_{\mathrm{chl}}=80 \mu \mathrm{M}$. However, adhesion of cells to the microfluidic channels was observed at the latter concentration which could start to limit the performance of the device. We therefore restricted all the data presented in this work to $c_{c h l}=40 \mu \mathrm{M}$ where no difficulties with the operation were noted over an operation period of several hours. Different strategies could be implemented for circumventing the problem of cell adhesion, such as treatment of the device surfaces. The increasing power output with the cell concentration suggests that flow-based operation can provide a simple approach to increase the power output of the devices, resulting in an advantage over systems where the delivered power is limited to the monolayer of the cells on the electrode.

Exoelectrogenic activity in wild-type photosynthetic organisms is not optimal for production of 
photocurrent, since some of the electrons generated are subsequently consumed by a series of electron sinks ${ }^{9}$. This is likely to reduce the power production. To overcome this limitation, we generated a Synechocystis mutant deficient in the main photosynthetic and respiratory electron sinks, the terminal oxidases, (cytochrome $c$ oxidase, quinol oxidase and the alternative respiratory terminal oxidase), and the flavodiiron complexes, Flv1/3 and Flv2/4 (Methods and Supplementary Figure 3$)^{24,37}$. We predicted that the absence of these electron sinks would lead to elevated exoelectrogenic activity and hence also to increased BPV power outputs. Indeed, the characterisation of the BPV with mutant cells showed that the power output was doubled compared to the wild-type cells at the same chlorophyll concentration $\left(c_{c h l}=40 \mu M\right)$ reaching $(44.6 \pm 1.6) \mathrm{nW}$ (Figure 4a,b).

In order to compare devices of different dimensions, current and power outputs are commonly normalised to the active surface area of the anode where the phototrophic cells are located ${ }^{9,32}$. The peak anodic power density for the described $\mu$-BPV can thus be estimated to be around $(0.27 \pm$ $0.03) \mathrm{W} \mathrm{m}^{-2}$ for the wild-type cells and $(0.54 \pm 0.05) \mathrm{W} \mathrm{m}^{-2}$ for the mutant cells $\left(c_{c h l}=40 \mu M\right.$ for both). To the best of our knowledge these values exceed those of previously demonstrated BPVs by two and a half fold and five-fold, respectively (Figure 4c; Supplementary Table 1).

\section{Continuous operation and scalability}

We have shown that while flowing through the device, the cells and their exoelectrogenic performance remained unaffected (Figure $2 b, c)$. However, during every cycle some fraction of the 
electron carrier molecules diffuses from the anodic area to the cathodic one, eventually leading to a decrease in its overall concentration in the anolyte. To show that this diffusion process does not affect the possibility to operate the devices continuously, we also included the electron carrier molecules $\left[\mathrm{Fe}(\mathrm{CN})_{6}\right]^{-3}$ in the catholyte such that there would be no net movement of the $\left[\mathrm{Fe}(\mathrm{CN})_{6}\right]^{-3}$ ions from one side to another. Crucially, this addition did not affect the device performance (Supplementary Figure 4). With the reduced electron carrier molecule $\left[\mathrm{Fe}(\mathrm{CN})_{6}\right]^{-4}$ eventually oxidising back to $\left[\mathrm{Fe}(\mathrm{CN})_{6}\right]^{-3}$ (Supplementary Figure 1a) the system never runs short of the oxidised electron carrier $\left[\mathrm{Fe}(\mathrm{CN})_{6}\right]^{-3}$, setting the basis for continuous operation.

We note that as opposed to previously described BPVs, where the charging and power delivery process occurred in a single compartment, for a spatially decoupled operation the area involved in the charging process can in general be different from the anode area. To allow for an effective comparison with non-biological PV systems, we set out to estimate the additional area that is required for the illumination process. By comparing the total amount of charged electron carrier that can be generated under a fixed illumination area to the area that is needed for its conversion to electrical current we concluded that the area required for illumination is slightly smaller but of a similar size (Supplementary Note 2). As there is no requirement for the power delivery area to be exposed to sunlight, the illumination unit can be for instance be positioned above it (Figure 4d). We further note that in this experiment the geometry of the illumination unit was not optimised; generation of larger quantities of the reduced electron carriers molecules is possible (e.g. by using a deeper vessel), and is thus not the limiting area in the scalability consideration. As such, comparison of power densities normalised by anode area is an effective measure for understanding the scalability 
(a)

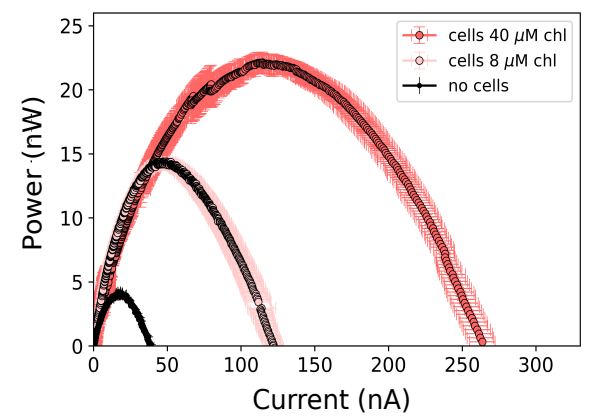

(c)

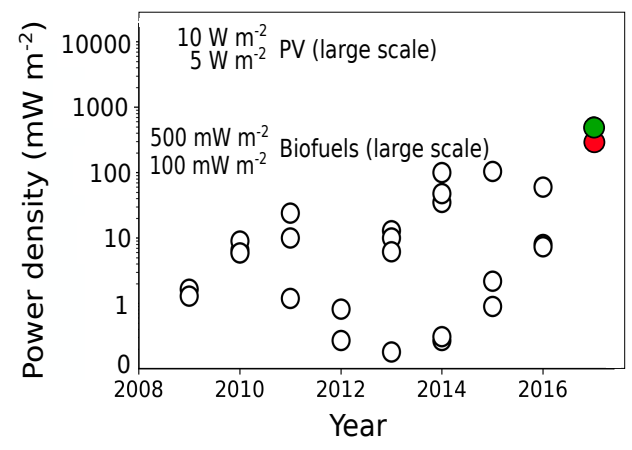

(b)

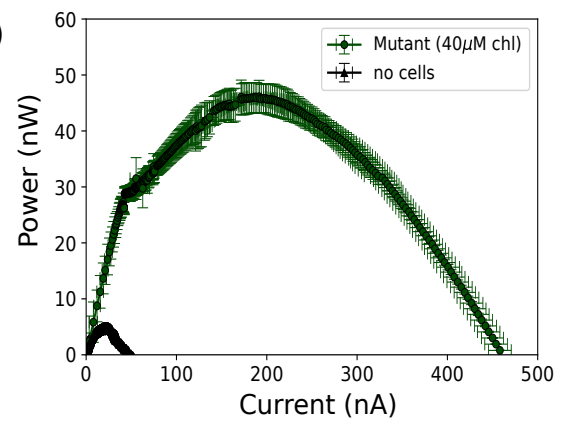

(d)

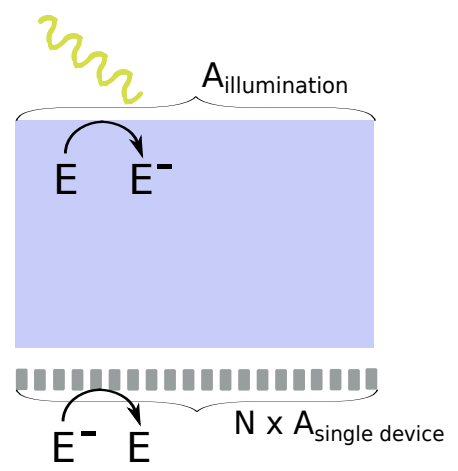

Figure 4: Dependence of the $\mu$-BPV performance on cell concentration and genotype, device configuration, and comparison with literature data. (a) An increase in the cell concentration yielded higher current and power outputs. (b) Using a mutant deficient in the main photosynthetic and respiratory electron sinks, the anodic power density of the BPV doubled compared to wild-type cells at the same concentration $\left(c_{c h l}=40 \mu M\right)$. (c) The obtained outputs are the highest BPV power densities to date - white markers correspond to previously described devices (Supplementary Table 1), red and green markers to the device described in this work with wild-type $((0.26 \pm$ $\left.0.03) \mathrm{W} \mathrm{m}^{-2}\right)$ and the mutant cells $\left((0.53 \pm 0.05) \mathrm{W} \mathrm{m}^{-2}\right)$, respectively. These values are similar to the power outputs of large scale biofuels and a factor of 10-20 lower than for large scale PVs ${ }^{38-40}$. (d) The areas required for the production of charged carrier and for its conversion to current were found to be of similar size. As there is no requirement to expose the delivery unit to light we propose placing it underneath the charging unit. 
of the system.

The current system used a platinum wire as cathode, given its previously proven catalytic activity for the re-generation of water. We found that the system yielded identical performance with the use of platinum-plated wire, which provides a promising approach for reducing the cost of the catalyst needed to below a cent for a thousand microfluidic chips (Supplementary Note 3 and Supplementary Figure 5). Additionally, non-platinum based materials have been shown to function effectively as the cathodic catalysts in microbial fuel cells ${ }^{41,42}$ and in BPVs ${ }^{43}$ providing an alternative strategy for reducing the cost of these systems.

\section{Conclusions}

The several-fold improvement over previously described devices achieved in this work combined with the advantages of BPVs, such as potentially cheap manufacturing and use of self-replicating catalysts, indicate that when scaled up via parallelisation, flow-BPVs could be a promising strategy for producing competitive analogues to synthetic photovoltaics (Figure 4c). Moreover even without parallelisation, the power outputs an individual device is sufficient for operating ultra-low power nanoelectronics, such as nanowire biosensors and their small size could allow them to be used to build self-sustaining nanosystems via on-chip integration ${ }^{31,44}$. The decoupling "division-ofunit-processes" strategy described here could also be applied to non-biological photovoltaics or to hybrid devices where conventional synthetic photovoltaic materials are employed in the charging process and an independently optimised electrocatalytic process in the electrochemical conversion 
unit.

In conclusion, we have described and demonstrated a flow-based BPV system where the charging and the power delivery units are spatially decoupled from another. In contrast to previously described devices, such decoupling provides temporal flexibility in power generation and the opportunity to independently optimise the geometry and the conditions needed for light harvesting and power delivery processes. In particular, the use of microscale channels in the power delivery unit enabled us to separate the anolyte and catholyte streams and dispense with the membranes, simplifying fabrication. Using this device, we have achieved a two and a half fold improvement over the highest power outputs recorded in any BPV using wild-type cells and a fivefold improvement using a novel strain deficient in several electron sinks. We envisage microscale devices to be a promising approach to optimise BPVs, thus opening a path towards fulfilling their potential as a cheap and environmentally friendly complement to non-biological photovoltaics.

\section{Methods}

Cell culture and growth. Wild-type Synechocystis sp. PCC $6803^{45}$ and recombinant strains were routinely cultured in BG-11 medium supplemented with $10 \mathrm{mM} \mathrm{NaHCO}{ }^{46}$ and maintained in sterile conditions at $30 \pm 2{ }^{\circ} \mathrm{C}$ under continuous moderate light of $40 \mu \mathrm{mol}$ photons $\mathrm{m}^{-2} \mathrm{~s}^{-1}$ and shaking at $160 \mathrm{rpm}$. A bench-top centrifuge (5000 rpm for 3 minutes) was used to concentrate the cells. The concentration of chlorophyll in samples was determined spectrophotometrically from the optical density values at $680 \mathrm{~nm}$ and $750 \mathrm{~nm}$ as described previously ${ }^{28}$. 
Generation of recombinant strains of Synechocystis sp. PCC 6803 Unmarked mutants of Synechocystis lacking Flv2 and Flv3 were constructed by disruption of $f l v 2$ and $f l v 3$, respectively, in the terminal oxidase mutant deficient strain via a two step homologous recombination protocol ${ }^{28}$. To generate marked mutants approximately $1 \mu \mathrm{g}$ of plasmids pFlv2-2 and pFlv3-2 (construction process described in Supplementary Note 4) were mixed with Synechocystis cells for 6 hours in liquid medium, followed by incubation on BG-11 agar plates for approximately 24 hours. An additional $3 \mathrm{~mL}$ of agar containing kanamycin was added to the surface of the plate followed by further incubation for approximately 1-2 weeks. Transformants were subcultured to allow segregation of mutant alleles. Segregation was confirmed by PCR using primers Flv2f/Flv2r, or Flv3f/Flv3r, which flank the deleted region. Generation of unmarked mutants was carried out as described previously ${ }^{47}$. To remove the $n p t 1 /$ sacRB cassette, mutant lines were transformed with $1 \mu \mathrm{g}$ of the markerless pFlv2-1 and pFlv3-1 constructs. Following incubation in BG-11 liquid medium for 4 days and on agar plates containing sucrose $(5 \% \mathrm{w} / \mathrm{v})$ for a further $1-2$ weeks, transformants were patched on kanamycin and sucrose plates. Sucrose resistant, kanamycin sensitive strains containing the unmarked deletion were confirmed by PCR using primers flanking the deleted region (Supplementary Figure 3).

BPV design and fabrication. The microfluidic devices were fabricated in poly(dimethylsiloxane) (PDMS; Dow Corning) through single, standard soft-lithography steps using SU-8 3025 photoresist (MicroChem) on a polished silicon wafer ${ }^{48}$. After removing the PDMS slab from the patterned silicon wafer access ports (two for fluid inlets, the fluid outlets and the anode, one for the cathode) were introduced by a UniCore hole punch ( $0.75 \mathrm{~mm}$ diameter, Harris). The surfaces of the 
channels were activated through oxygen plasma and sealed with microscope glass slides (Thermo Scientific).

The flow channel (Figure 2a) was designed to be $250 \mu \mathrm{m}$ in width and $6500 \mu \mathrm{m}$ in length and was fabricated to a height of $25 \mu \mathrm{m}$. These dimensions were chosen so that neither the cyanobacterial cells nor the molecules of the electron carrier could diffuse all the way to the cathode before exiting the channel. The flow channel was separated from the anode by an array of $25 \mu \mathrm{m}$ wide PDMS pillars separated by $25 \mu \mathrm{m}$. This allowed for the insertion of molten anode material (Indalloy 19 (51\% In, 32.5\% Bi, 16.5\% In), Conro Electronics) at $79{ }^{\circ} \mathrm{C}$ that solidified upon removal from the hot-plate and yielded self-aligned electrode walls ${ }^{49,50}$. The cathode was constructed by inserting a strip of $100 \mu \mathrm{m}$ diameter Pt wire (AlfaAesar) through polyethylene tubing (Smiths Medical; 800/100/120) sealed by an epoxy glue from both ends with approximately $1 \mathrm{~mm}$ metal left outside the tubing. This allowed direct contact of the metal with the fluids flowing in the BPV when placing the cathode in the BPV via its designated access port. Last, copper wires were soldered to both the cathode and the anode, and alligator clamps were used to connect the electrodes to the potentiometer (BPV characterisation).

BPV operation Before injecting the biological material into a device, the background current was characterised by injecting the cell medium containing an electron carrier $\left(30 \mathrm{mM} \mathrm{K}_{3}\left[\mathrm{Fe}(\mathrm{CN})_{6}\right]\right)$ via both the cathodic and anodic inlets using $1 \mathrm{~mL}$ and $0.25 \mathrm{~mL}$ Hamilton glass syringes, respectively. The syringes were connected to the microfluidic access ports via polyethylene tubing (Smiths Medical; 800/100/120) and 27-gauge needles and the flows were controlled via neMESYS syringe pumps (Cetoni $\mathrm{GmbH})$. The performance of the BPV was then characterised in its biologically 
loaded form by injecting a mixture of $30 \mathrm{mM} \mathrm{K}_{3}\left[\mathrm{Fe}(\mathrm{CN})_{6}\right]$ and cyanobacterial cells at a specified concentration into the anodic chamber. Before injection, $1 \mathrm{~mL}$ of the mixture was illuminated for 3 hours under red light (Maplin Strip RGB kit) at a constant output of $30 \mu \mathrm{mol}$ photons $\mathrm{m}^{-2} \mathrm{~s}^{-1}$ to reduce the $\mathrm{K}_{3}\left[\mathrm{Fe}(\mathrm{CN})_{6}\right]$ by the exoelectrogenic electrons released by the photosynthesising cells. The light intensity was measured at the location of the sample using a quantum sensor uniformly detecting photosynthetically active radiation between 400 and $700 \mathrm{~nm}$ (Skye Instruments) and the solution was spread over a circular area with a diameter of $1.0 \mathrm{~cm}$.

BPV characterisation The performance of the BPV was characterised by applying a linear voltage sweep (Autolab PGSTAT 12) across the positive and negative terminals. This was first applied to the biologically non-loaded form of the BPV and then the biologically loaded form. After a stable terminal voltage value had been reached the applied voltage was varied from $800 \mathrm{mV}$ to $0 \mathrm{mV}$ at a rate of $2 \mathrm{mV} \mathrm{s}^{-1}$ and the current output recorded at each voltage. The device was kept at $22 \pm 2{ }^{\circ} \mathrm{C}$ throughout the characterisation process. The delivered power was derived from the relationship

$$
P=U \times I
$$

To allow comparison with existing BPVs, the power output was normalised per unit area of the anode:

$$
A_{\text {anode }}=\frac{1}{2} \times 6.5 \mathrm{~mm} \times 0.025 \mathrm{~mm}=0.08 \mathrm{~mm}^{2}
$$

where the fraction $\frac{1}{2}$ arises from only half of the anode area being available for electron transfer and the other half being covered by the PDMS pillars. 
The data that support the plots within this paper and other findings of this study are available from the corresponding authors upon request.

\section{References}

1. International Energy Agency, Solar Energy Perspectives (2011).

2. British Petroleum, BP Statistical Review of World Energy (2015).

3. Green, M. A. Commercial progress and challenges for photovoltaics. Nature Energy 1, 15015 (2016).

4. Crabtee, G. W. \& Lewis, N. S. Solar energy conversion. Physics Today 60, 37-42 (2007).

5. Lewis, N. S. \& Nocera, D. G. Powering the planet: Chemical challenges in solar energy utilization. Proceedings of the National Academy of Sciences 103, 15729-15735 (2006).

6. Tao, C. S., Jiang, J. \& Tao, M. Natural resource limitations to terawatt-scale solar cells. Solar Energy Materials and Solar Cells 95, 3176-3180 (2011).

7. Peter, L. M. Towards sustainable photovoltaics: the search for new materials. Philosophical Transactions of the Royal Society of London A: Mathematical, Physical and Engineering Sciences 369, 1840-1856 (2011).

8. Mazzio, K. A. \& Luscombe, C. K. The future of organic photovoltaics. Chemical Society Reviews 44, 78-90 (2014). 
9. McCormick, A. J. et al. Biophotovoltaics: oxygenic photosynthetic organisms in the world of bioelectrochemical systems. Energy Environ. Sci. 8, 1092-1109 (2015).

10. Hasan, K. et al. Photo-electrochemical communication between cyanobacteria (Leptolyngbia sp.) and osmium redox polymer modified electrodes. Physical Chemistry Chemical Physics 16, 24676-24680 (2014).

11. Hasan, K. et al. Photoelectrochemical wiring of paulschulzia pseudovolvox (algae) to osmium polymer modified electrodes for harnessing solar energy. Advanced Energy Materials $\mathbf{5}$ (2015).

12. McCormick, A. J. et al. Photosynthetic biofilms in pure culture harness solar energy in a mediatorless bio-photovoltaic cell (BPV) system. Energy \& Environmental Science 4, 4699_ 4709 (2011).

13. Hambourger, M. et al. Biology and technology for photochemical fuel production. Chemical Society Reviews 38, 25-35 (2009).

14. Tanaka, K., Tamamushi, R. \& Ogawa, T. Bioelectrochemical fuel-cells operated by the cyanobacterium, Anabaena variabilis. Journal of Chemical Technology and Biotechnology 35, 191-197 (1985).

15. Zou, Y., Pisciotta, J., Billmyre, R. B. \& Baskakov, I. V. Photosynthetic microbial fuel cells with positive light response. Biotechnology and Bioengineering 104, 939-946 (2009). 
16. Bradley, R. W., Bombelli, P., Rowden, S. J. \& Howe, C. J. Biological photovoltaics: intraand extra-cellular electron transport by cyanobacteria. Biochemical Society Transactions $\mathbf{4 0 ,}$ 1302-1307 (2012).

17. Bombelli, P. et al. Quantitative analysis of the factors limiting solar power transduction by Synechocystis sp. PCC 6803 in biological photovoltaic devices. Energy \& Environmental Science 4, 4690-4698 (2011).

18. Squires, T. M. \& Quake, S. R. Microfluidics: Fluid physics at the nanoliter scale. Reviews of Modern Physics 77, 977 (2005).

19. Ferrigno, R., Stroock, A. D., Clark, T. D., Mayer, M. \& Whitesides, G. M. Membraneless vanadium redox fuel cell using laminar flow. Journal of the American Chemical Society 124, 12930-12931 (2002).

20. Wang, H.-Y., Bernarda, A., Huang, C.-Y., Lee, D.-J. \& Chang, J.-S. Micro-sized microbial fuel cell: a mini-review. Bioresource Technology 102, 235-243 (2011).

21. Yang, J., Ghobadian, S., Goodrich, P. J., Montazami, R. \& Hashemi, N. Miniaturized biological and electrochemical fuel cells: challenges and applications. Physical Chemistry Chemical Physics 15, 14147-14161 (2013).

22. Kjeang, E. et al. High-performance microfluidic vanadium redox fuel cell. Electrochimica Acta 52, 4942-4946 (2007). 
23. Ren, H., Torres, C. I., Parameswaran, P., Rittmann, B. E. \& Chae, J. Improved current and power density with a micro-scale microbial fuel cell due to a small characteristic length. Biosensors and Bioelectronics 61, 587-592 (2014).

24. Lea-Smith, D. J., Vasudevan, R. \& Howe, C. J. Generation of marked and markerless mutants in model cyanobacterial species. JoVE (Journal of Visualized Experiments) e54001 (2016).

25. Zhang, P. et al. Operon flv4-flv2 provides cyanobacterial photosystem II with flexibility of electron transfer. The Plant Cell 24, 1952-1971 (2012).

26. Brody, J. P. \& Yager, P. Diffusion-based extraction in a microfabricated device. Sensors and Actuators A: Physical 58, 13-18 (1997).

27. Kamholz, A. E., Weigl, B. H., Finlayson, B. A. \& Yager, P. Quantitative analysis of molecular interaction in a microfluidic channel: the T-sensor. Analytical Chemistry 71, 5340-5347 (1999).

28. Lea-Smith, D. J. et al. Thylakoid terminal oxidases are essential for the cyanobacterium Synechocystis sp. PCC 6803 to survive rapidly changing light intensities. Plant Physiology 162, 484-495 (2013).

29. Zhao, F., Slade, R. C. \& Varcoe, J. R. Techniques for the study and development of microbial fuel cells: an electrochemical perspective. Chemical Society Reviews 38, 1926-1939 (2009).

30. Logan, B. E. et al. Microbial fuel cells: methodology and technology. Environmental Science \& Technology 40, 5181-5192 (2006). 
31. Qian, F., Baum, M., Gu, Q. \& Morse, D. E. A $1.5 \mu$ l microbial fuel cell for on-chip bioelectricity generation. Lab on a Chip 9, 3076-3081 (2009).

32. Bombelli, P., Müller, T., Herling, T. W., Howe, C. J. \& Knowles, T. P. A high power-density, mediator-free, microfluidic biophotovoltaic device for cyanobacterial cells. Advanced Energy Materials 5, e1401299 (2015).

33. Kjeang, E., Djilali, N. \& Sinton, D. Microfluidic fuel cells: A review. Journal of Power Sources 186, 353-369 (2009).

34. Shaegh, S. A. M., Nguyen, N.-T. \& Chan, S. H. A review on membraneless laminar flow-based fuel cells. International Journal of Hydrogen Energy 36, 5675-5694 (2011).

35. Li, Z., Zhang, Y., LeDuc, P. R. \& Gregory, K. B. Microbial electricity generation via microfluidic flow control. Biotechnology and Bioengineering 108, 2061-2069 (2011).

36. Müller, T. et al. Particle-based simulations of steady-state mass transport at high Péclet numbers. arXiv preprint arXiv:1510.05126 (2015).

37. Lea-Smith, D. J., Bombelli, P., Vasudevan, R. \& Howe, C. J. Photosynthetic, respiratory and extracellular electron transport pathways in cyanobacteria. Biochimica et Biophysica Acta (BBA)-Bioenergetics 1857, 247-255 (2016).

38. MacKay, D. J. C. Solar energy in the context of energy use, energy transportation and energy storage. Phil. Trans. R. Soc. A 371, 20110431 (2013). 
39. Dabiri, J. O. et al. A new approach to wind energy: opportunities and challenges. In AIP Conference Proceedings, vol. 1652, 51-57 (AIP, 2015).

40. De Castro, C., Mediavilla, M., Miguel, L. J. \& Frechoso, F. Global solar electric potential: A review of their technical and sustainable limits. Renewable and Sustainable Energy Reviews 28, 824-835 (2013).

41. HaoYu, E., Cheng, S., Scott, K. \& Logan, B. Microbial fuel cell performance with non-pt cathode catalysts. Journal of Power Sources 171, 275-281 (2007).

42. Kakarla, R. \& Min, B. Photoautotrophic microalgae scenedesmus obliquus attached on a cathode as oxygen producers for microbial fuel cell (mfc) operation. International Journal of Hydrogen Energy 39, 10275-10283 (2014).

43. Schneider, K., Thorne, R. J. \& Cameron, P. J. An investigation of anode and cathode materials in photomicrobial fuel cells. Phil. Trans. R. Soc. A 374, 20150080 (2016).

44. Qian, F. \& Morse, D. E. Miniaturizing microbial fuel cells. Trends in biotechnology 29, 62-69 (2011).

45. Williams, J. G. Construction of specific mutations in photosystem II photosynthetic reaction center by genetic engineering methods in Synechocystis 6803. Methods in Enzymology 167, 766-778 (1988).

46. Castenholz, R. W. Culturing methods for cyanobacteria. Methods in Enzymology 167, 68-93 (1988). 
47. Lea-Smith, D. J., Vasudevan, R. \& Howe, C. J. Generation of Marked and Markerless Mutants in Model Cyanobacterial Species. Journal of Visualized Experiments 1-11 (2016).

48. Duffy, D. C., McDonald, J. C., Schueller, O. J. \& Whitesides, G. M. Rapid prototyping of microfluidic systems in poly (dimethylsiloxane). Analytical Chemistry 70, 4974-4984 (1998).

49. So, J.-H. \& Dickey, M. D. Inherently aligned microfluidic electrodes composed of liquid metal. Lab on a Chip 11, 905-911 (2011).

50. Herling, T. et al. Integration and characterization of solid wall electrodes in microfluidic devices fabricated in a single photolithography step. Applied Physics Letters 102, 184102 (2013).

Acknowledgements The research leading to these results has received funding from the Engineering and Physical Sciences Research Council (K.L.S., T.P.J.K.), the Leverhulme Trust (P.B., C.J.H., T.P.J.K.; RPG2015-393), the European Research Council under the European Union's Seventh Framework Programme (FP7/2007-2013) through the ERC grant PhysProt (agreement n 337969), the Biotechnology and Biological Sciences Research Council (T.P.C.; BB/J014540/1), the Environmental Services Association Education Trust (D.J.L-S) and the EnAlgae consortium (P.B., C.J.H.). The authors thank Lewis Lea for help in constructing Figure 1.

Competing Interests The authors declare that they have no competing financial interests.

Correspondence Correspondence and requests for materials should be addressed to Tuomas P. J. Knowles (email: tpjk2@cam.ac.uk) and Christopher Howe (email: ch26@cam.ac.uk)who contributed equally to this 
work. 


\section{Enhancing power density of biophotovoltaics by decou- pling storage and power delivery - Supplementary Materi- als}

Kadi L. Saar ${ }^{1}$, Paolo Bombelli ${ }^{1,2}$, David J. Lea-Smith ${ }^{2,3}$, Toby Call ${ }^{2}$, Eva-Mari Aro ${ }^{4}$, Thomas

Müller $^{1,5}$, Christopher J. Howe ${ }^{2} \&$ Tuomas P. J. Knowles ${ }^{1,6}$

${ }^{1}$ Department of Chemistry, University of Cambridge, Lensfield Road, Cambridge CB2 1EW, UK

${ }^{2}$ Department of Biochemistry, University of Cambridge, Tennis Court Road, Cambridge CB2 1QW, UK

${ }^{3}$ Current address: School of Biological Sciences, University of East Anglia, Norwich Research Park, Norwich NR4 7TJ, UK

${ }^{4}$ Laboratory of Molecular Plant Biology, Department of Biochemistry, University of Turku, Turku, FI-20014, Finland

${ }^{5}$ Fluidic Analytics Limited, Cambridge, UK

${ }^{6}$ Cavendish Laboratory, Department of Physics, University of Cambridge, J J Thomson Ave, Cambridge CB3 OHE, UK 
(a)

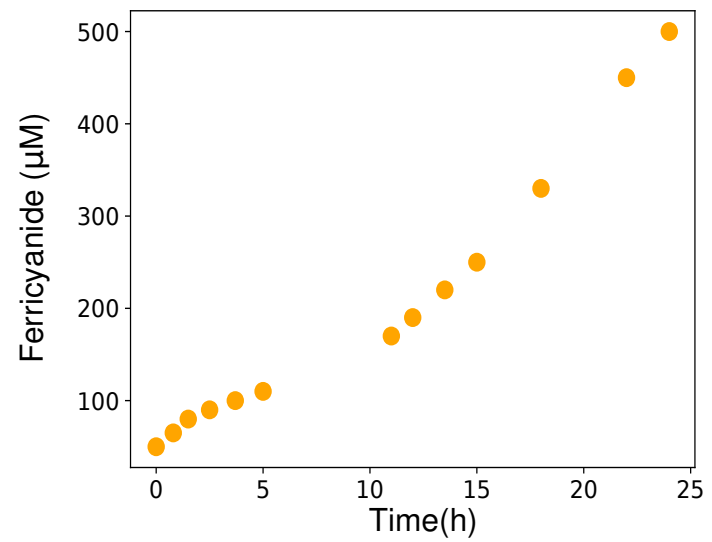

(b)

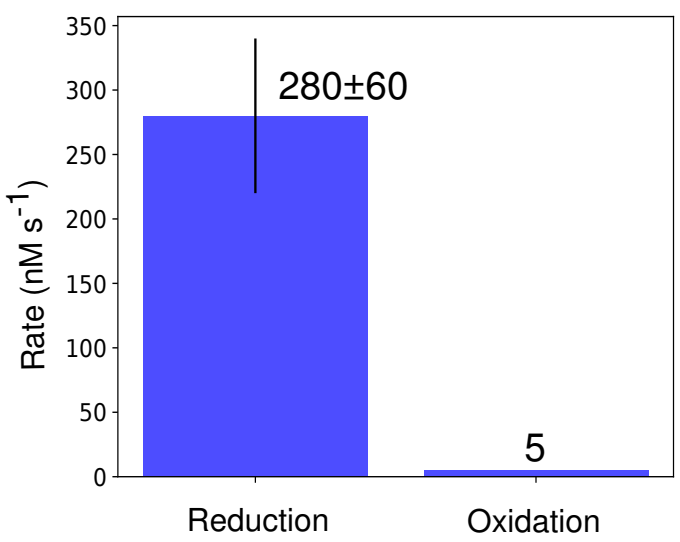

Supplementary Figure 1: (a) Ferrocyanide $\left(\left[\mathrm{Fe}(\mathrm{CN})_{6}\right]^{-4}\right)$ oxidation to ferricyanide $\left(\left[\mathrm{Fe}(\mathrm{CN})_{6}\right]^{-3}\right)$ in air over time. (b) The rate of the oxidation reaction can be seen to be significantly slower than the rate of ferricyanide $\left(\left[\mathrm{Fe}(\mathrm{CN})_{6}\right]^{-3}\right)$ reduction to ferrocyanide $\left(\left[\mathrm{Fe}(\mathrm{CN})_{6}\right]^{-4}\right)$ by the exoelectrogenic electrons released by the wild type photosynthetic cells in this study (average of $n=3$ repeats, error bars correspond to standard deviations). 
(a)

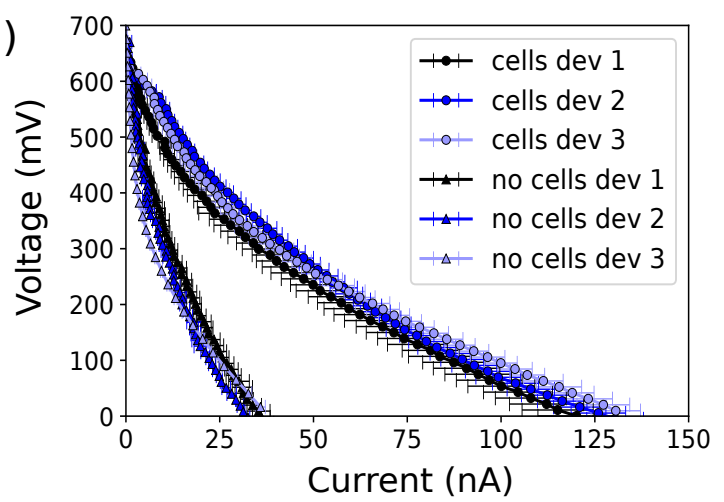

(b)

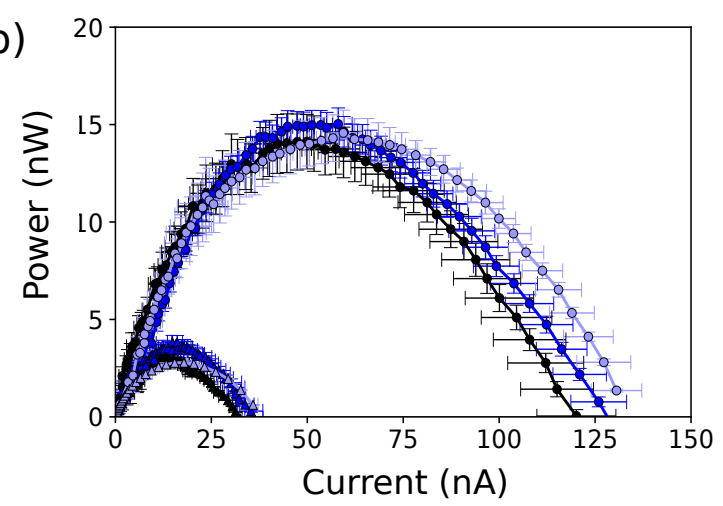

Supplementary Figure 2: A typical polarisation (a) and power (b) curve for the fabricated $\mu$-BPV at a flow rate of $Q_{\text {total }}=20 \mu \mathrm{L} \mathrm{h}^{-1}$ (average of $n=3$ repeats in three individually fabricated devices dev1-3; error bars correspond to standard deviations) with wild type cells (circles) at chlorophyll concentration of $c_{c h l}=8 \mu M$ and without cells (triangles). 
(a)

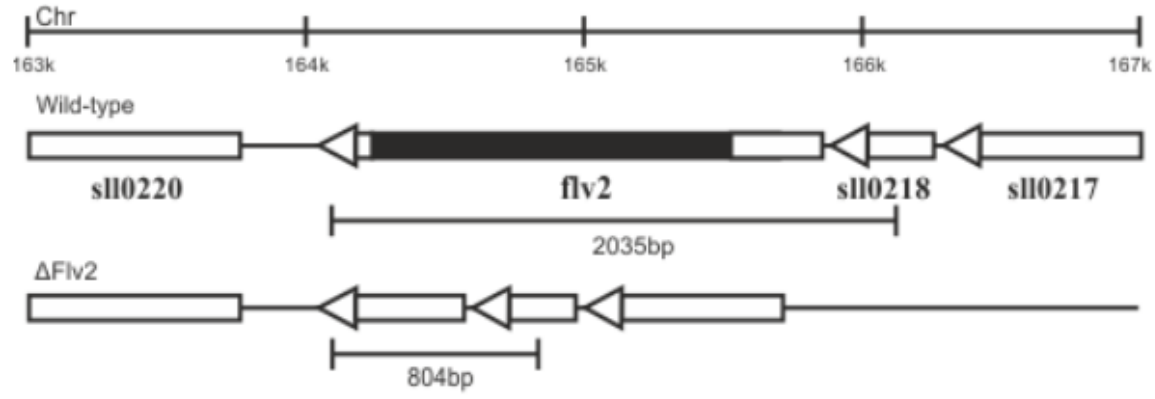

(b)
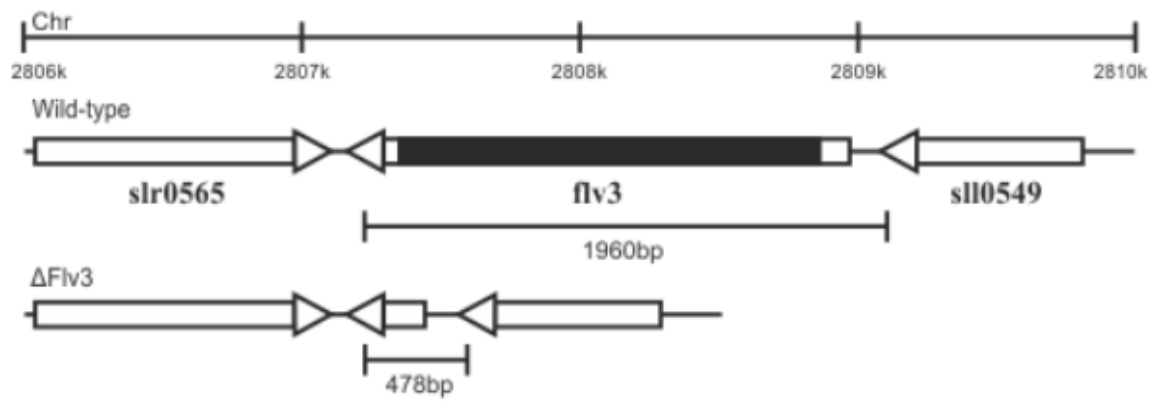

(c)

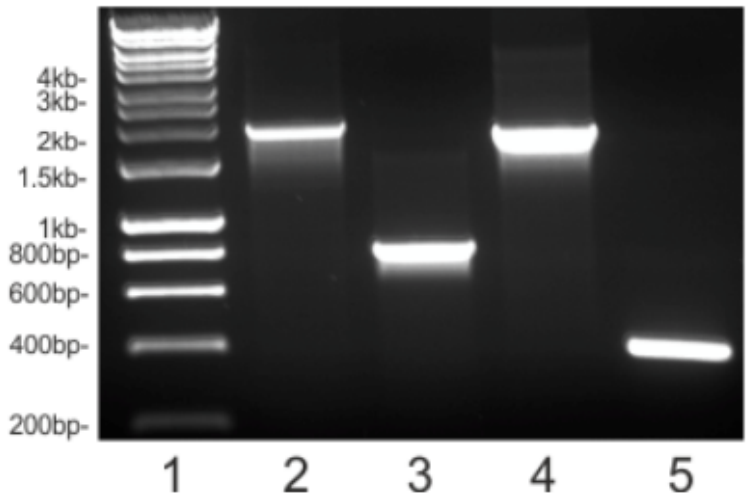

Supplementary Figure 3: Schematic representations of locus location in the Synechocystis sp. PCC6803 genome (top) and the wild type (middle) and unmarked knockouts (bottom) profiles expected in (a) $\Delta$ Flv2 and (b) $\Delta$ Flv3 strains following amplification with primers flanking the deleted sequence. Regions deleted in the mutant strains are shaded in black. (c) Amplification of genomic DNA in wild type cells (lane 2) and $\Delta F l v 2$ (lane 3) using Flv2f and Flv2r primers; in wild type (lane 4) and Flv3 (lane 5) using Flv3f and Flv3r primers. Markers are in Lane 1. 
(a)

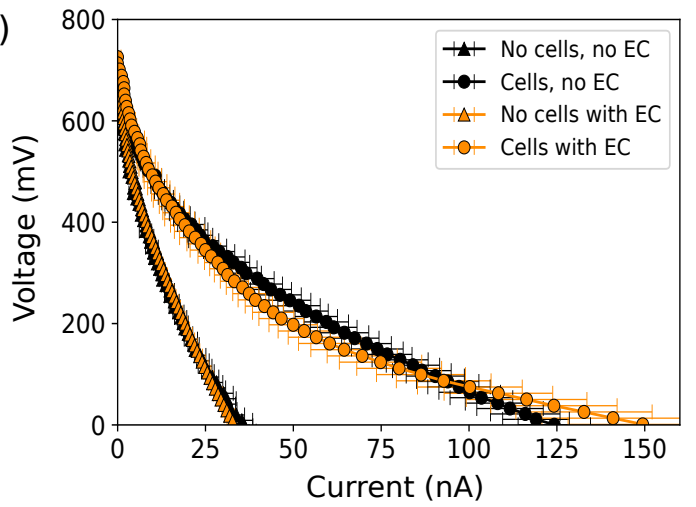

(b)

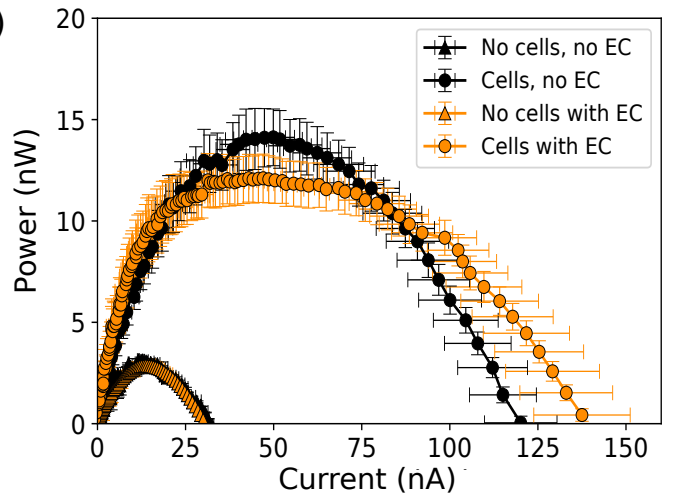

Supplementary Figure 4: The device performance was studied by including ferricyanide (30 mM) also in the catholyte as this ensures that the amount of electron carrier in the anolyte remains unchanged when the cells are circulated. The recorded (a) polarisation and (b) power curves (average of $n=3$ repeats, error bars correspond to standard deviations) were similar to those recorded with no ferricyanide in the cathodic chamber (Figure 2b in Main Text; both wild type cells at $\mathrm{c}_{\mathrm{chl}}=8 \mu \mathrm{M}$ ) enabling the device to be operated in recirculating mode. 
(a)

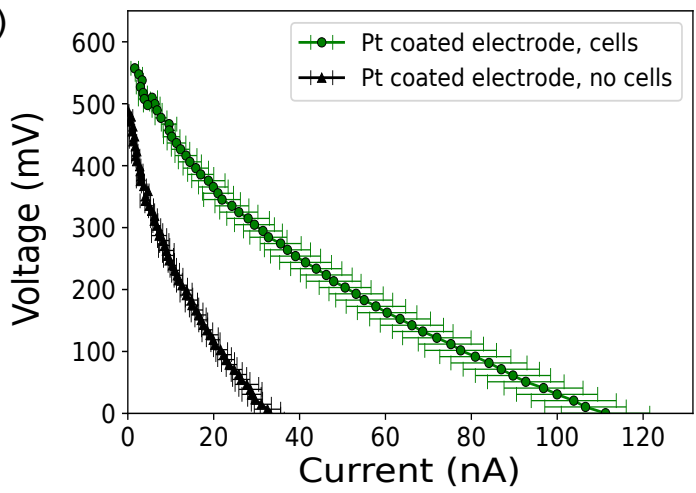

(b)

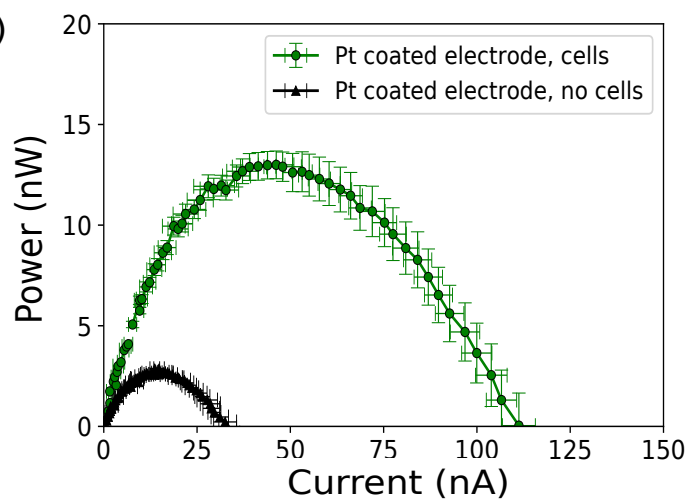

Supplementary Figure 5: (a) Polarisation and (b) power curves when a platinum plated wire was used as a cathode instead of a platinum wire (experiments performed with wild type cells at $\mathrm{c}_{\mathrm{chl}}=8 \mu \mathrm{M}$; average of $n=3$ repeats, error bars correspond to standard deviations). The recorded power output was not significantly different from that observed when using platinum wire while the cost of the electrode is reduced by many orders of magnitude. 
Supplementary Table 2. Sequence of primers used in this study.*

\begin{tabular}{|l|l|}
\hline Primer name & Sequence (5 to 3) \\
\hline Flv2leftfor & GATCGAATTCGCGTTGCTGGTTATCCAAGT \\
Flv2leftrev & GATCGAGCTCGGAGTGTACGGCGTCATTTT \\
Flv2rightfor & GATCGGATCCAAGCTGGGCTAAATCCAAA \\
Flv2leftrev & GCATICTAGAGCACCATACAAACTGGACGA \\
Flv3leftf & GATCGAATTCGGTGTTCAGCGGTTACTTA \\
Flv3leftr & GATCGGATCCGCGCTAGCCTATTTGGAGTG \\
Flv3rightf & GATCGGATCCCAGTCCAAAGAACGGATA \\
Flv3leftr & GCATTCTAGAGGGAAAAGGGTGGGATGTAT \\
Flv2for & GCCGTTGGGTTGTAACACTT \\
Flv2rev & GTTTCCATCGGAGTTGCAGT \\
Flv3for & GCGGTTTGATTGCAGTTTTT \\
Flv3rev & GGGGAAAGCATCATGTATCG
\end{tabular}

* Restriction endonuclease sites introduced into the primer are underlined. 


\section{Supplementary Note 1 - Energy dissipation in the $\mu$-BPV}

When the fluids are flowing in the BPV they lose energy due to friction (Figure 1b in Main Text). This energy dissipation can be estimated to be:

$$
\begin{aligned}
P_{\text {friction }} & =[\Delta P \times \text { Area }] \times v \\
& =R_{h} \times Q_{\text {total }} \times(w \times h) \times \frac{Q_{\text {total }}}{w \times h} \\
& =R_{h} \times Q_{\text {total }^{2}}^{2}
\end{aligned}
$$

where the hydrodynamic resistance of the channel $R_{h}$ can be estimated as described by Mortensen et al. ${ }^{1}$.

Specifically, at a flow rate $Q_{\text {total }}=20 \mu \mathrm{L} \mathrm{h}^{-1}$ the pressure drop can be estimated to be $\Delta P=$ $110 \mathrm{~Pa}$ and the associated frictional loss $P_{\text {friction }} \approx 0.5 \mathrm{nW}$ which is a small fraction of the power generated by the device at this flow rate $(14.0 \mathrm{nW}$ for wild type cells at the lowest used cell concentration and $22.2 \mathrm{nW}$ at the highest concentration). The resulting temperature increase from the dissipated power is negligible.

Similar estimates for the dissipated energy $P_{\text {friction }}$ are obtained when the pressure drop $\Delta P \mathrm{~s}$ instead approximated using the Darcy-Weisbach equation:

$$
\Delta P=L \times \frac{f_{D}}{d_{H}} \times \frac{\rho \times v^{2}}{2}
$$

where the friction factor $f_{D}$ is estimated from:

$$
f_{D}=\frac{64}{R e}
$$


and the hydraulic diameter $d_{h}$ from:

$$
d_{h}=\frac{4 \times A}{P}=\frac{4 \times A}{2 \times(w+h)}
$$

The pressure drop was also measured experimentally (Elveflow microfluidic sensor OB1) and the measured result, 1 mbar agreed with the estimates. We further note the actual loss could be up to $50 \%$ higher due to non-ideal operation of pumps (typical efficiencies range from $65 \%$ to $90 \%$ ) but is nevertheless a small fraction of the total power outputs at the typical operating conditions used in this work (Figure 3b in Main Text).

\section{Supplementary Note 2 - Comparison of the areas involved in the illumination and in the power harvesting processes}

In order to compare the areas involved in the illumination and the power harvesting process we illuminated $V=3 \mathrm{~mL}$ of wild type cell suspension under a fixed area of $\mathrm{A}_{\text {illumination }}=1 \mathrm{~cm}^{2}$ and recorded the amount of the charged up electron carrier produced. We determined the rate of ferrocyanide generation by the cells under typical light conditions ${ }^{4}$ of $2000 \mu \mathrm{E} \mathrm{m}^{-2} \mathrm{~s}^{-1}$ to be (280 $\pm 60) \mathrm{nM} \mathrm{s}^{-1}$ (average of $n=3$ repeats; Supplementary Figure 1b). The current flowing in our $\mu$-BPV at its peak power output, $50 \mathrm{nA}$ (Figure 2b in Main Text), corresponds to ferrocyanide

reduction rate of $1.3 \mathrm{pmol} \mathrm{s}^{-1}$. We can therefore estimate that a total of $\mathrm{N}=\frac{0.28 \mu \mathrm{M} \mathrm{s}^{-1} \cdot 3 \mathrm{~mL}}{1.3 \mathrm{pmol} \mathrm{s}^{-1}}=$ 1620 channels would be required for converting all the charged up electron carrier into current. With the area of a single channel being $\mathrm{A}_{\text {anode }}=0.08 \mathrm{~mm}^{2}$, the total area required for harvesting 
the power is $\mathrm{A}_{\text {power delivery }}=1.3 \mathrm{~cm}^{2}$, indicating that the two areas are of a similar size.

\section{Supplementary Note 3 - The choice of suitable catalyst for the cathode}

The current system used a platinum wire as cathode, given its previously proven catalytic activity for the re-generation of water. We tested whether a platinum-plated wire could be used instead of a platinum wire, and found that whilst this reduced the total cost of the system the maximum power output of the system was not significantly affected (Supplementary Figure 5). Platinumplated wires are available at a price of $\$ 0.02$ per hundred metres $(\$ 35 / \mathrm{kg})$, using a conservative estimate of a few centimetres of wire per chip the cost of the amount of wire needed for a thousand microfluidic chips is below a cent which is significantly smaller than the cost of a microfluidic chip itself.

\section{Supplementary Note 4 - Plasmid construction for the generation of electron transport chain} mutants

All the primers used in this study are listed in Supplementary Table 2. The genome sequence of Synechocystis sp. PCC6803 was consulted via Cyanobase (http://genome.kazusa.or.jp/cyanobase) for primer design ${ }^{2}$. Gene deletion of $f l v 2$ was performed by amplifying a $912 \mathrm{bp}$ fragment upstream of $f l v 2$ using primers Flv2leftfor and Flv2leftrev and a 972bp fragment downstream of $f v 2$ using 
primers Flv2rightfor and Flv2rightrev, followed by insertion of the respective fragments into the SacI/EcoRI and XbaI/BamHI sites of pUC19 to generate pFlv2-1. Gene deletion of $f l v 3$ was performed by amplifying a 918bp fragment upstream of $f v 3$ using primers Flv3leftfor and Flv3leftrev and a 964bp fragment downstream of $f l v 3$ using primers Flv3rightfor and Flv3rightrev, followed by insertion of the respective fragments into the $S a c \mathrm{I} / E c o$ RI and $X b a \mathrm{I} / B a m \mathrm{HI}$ sites of pUC19 to generate Flv3-1. The BamHI digested $n p t 1 / s a c \mathrm{RB}$ cassette from pUM24Cm was inserted into the BamHI site between the upstream and downstream fragments in pFlv2-1 and pFlv3-1 to generate pFlv2-2 and pFlv3-2 respectively ${ }^{3}$. PCR amplification was performed by standard procedures using Phusion high fidelity DNA polymerase (NEB).

\section{Supplementary References}

1. Mortensen, N. A., Okkels, F. \& Bruus, H. Reexamination of Hagen-Poiseuille flow: Shape dependence of the hydraulic resistance in microchannels. Physical Review E - Statistical, Nonlinear, and Soft Matter Physics 71, 1-4 (2005).

2. Kaneko, T. et al. Sequence analysis of the genome of the unicellular cyanobacterium Synechocystis sp. strain PCC6803. II. Sequence determination of the entire genome and assignment of potential protein-coding regions. DNA research : an international journal for rapid publication of reports on genes and genomes 3, 109-136 (1996).

3. Ried, J. L. \& Collmer, A. An nptI-sacB-sacR cartridge for constructing directed, unmarked 
mutations in gram-negative bacteria by marker exchange-eviction mutagenesis. Gene 57, 239246 (1987).

4. Hall, D. O. \& Rao, K. K. Photosynthesis - Sixth edition (Cambridge University Press, 1999). 